\title{
Management of hernial orifices in robotic inguinal hernia repair
}

\author{
Johannes Baur, Michaela Ramser, Ulrich A. Dietz \\ Department of Visceral, Vascular and Thoracic Surgery, Kantonsspital Olten, Olten 4600, Switzerland. \\ Correspondence to: Dr. Johannes Baur, Department of Visceral, Vascular and Thoracic Surgery, Kantonssppital Olten, Baslerstr. \\ 150, Olten 4600, Switzerland. E-mail: johannes.baur@spital.so.ch
}

How to cite this article: Baur J, Ramser M, Dietz UA. Management of hernial orifices in robotic inguinal hernia repair. Miniinvasive Surg 2021;5:27. https://dx.doi.org/10.20517/2574-1225.2021.28

Received: 26 Feb 2021 First Decision: 35 Mar 2021 Revised: 1 Apr 2021 Accepted: 13 Apr 2021 Available online: 6 Jun 2021

Academic Editor: William W. Hope Copy Editor: Yue-Yue Zhang Production Editor: Yue-Yue Zhang

\begin{abstract}
The development of a postoperative seroma after endoscopic transabdominal (TAPP) or extraperitoneal (TEP) groin repair is a frequent problem. Although seromas are usually only mildly symptomatic, the swelling that develops postoperatively often causes patients to feel insecure and worried. In the literature some technical approaches to reduce the incidence of postoperative seroma are described. This technical note deals with the authors' approach in the management of large medial and lateral hernial orifices during robotic r-TAPP procedures using DaVinci Xi technology with the aim of seroma prophylaxis.
\end{abstract}

Keywords: Inguinal hernia, robotic surgery, hernial orifices, seroma, barbed suture, TISSEEL, fibrin sealant

\section{INTRODUCTION}

The postoperative occurrence of seroma is a challenge after inguinal hernia repair and often leads to insecurity and concern of patients, who may misinterpret the postoperative swelling as an early hernia recurrence, a finding also known as pseudo-recurrence. In some cases, the seroma can also affect the nerves of the groin region and lead to increased postoperative pain or even the development of a chronic pain syndrome ${ }^{[1]}$. Despite the usual spontaneous remission of seromas in the course of a few weeks, it is reasonable to find strategies to reduce the risk of postoperative seroma formation. The reported incidence of postoperative seroma varies between $0.5 \%-12.2 \%{ }^{[2]}$. 
Both, the morphology of the hernia and the surgical technique have been shown to influence postoperative seroma formation. In a large register-based study with more than 20,000 groin hernia patients treated by transabdominal pre-peritoneal (TAPP) laparoscopic techniques, multivariate analysis indicated that medial hernias and large hernias (EHS type 2 and 3 ) were associated with a significantly higher occurrence rate of seroma. Further, the method of mesh fixation was shown to have an impact; meshes fixed with fibrin glue had higher seroma rates than meshes fixed with staples. The risk of seroma was lowest if the mesh was not fixed at all $^{[3]}$. There are some specific surgical measures intended to prevent seroma formation; an overview of the outcome of some of these techniques is presented in the systematic review by Li et al. ${ }^{[4]}$.

Generally, 2 areas of interest for prophylactic measures regarding seroma formation have been investigated. First, the medial hernial orifice with the weakened transverse fascia in large hernias can be targeted. A low rate of postoperative seroma has been achieved both by tightening the transverse fascia with an endoloop ${ }^{[5]}$ as well as with a V-Loc suture ${ }^{[6]}$. Secondly, in the area of the lateral hernial orifices, the application of fibrin glue spray into the inguinal canal has been investigated, showing a reduction in size and rate of postoperative seromas $s^{[7]}$. Although the placement of surgical drains may reduce the risk of postoperative seroma formation, its routine use is not recommended by the European Hernia Society ${ }^{[2]}$.

The quality of all available studies on the management of hernia orifices during endoscopic inguinal hernia repair is limited due to the small number of patients examined. It can therefore be assumed that the techniques presented here have not yet been widely adopted. The recent introduction of robotics into endoscopic inguinal hernia repair promises large advantages, mainly the easiness to suture, the unprecedented accuracy and precision of the instruments use and the advantages of immersion view. First results pointing in this direction have recently been presented in a study where a suture retraction of the transverse fascia was performed in a total of 67 robotic TAPP (rTAPP) procedures, with no seroma nor other complication being recorded in the 30 -day follow-up ${ }^{[8]}$.

The use of DaVinci technology makes it possible to perform not only precise, nerve-sparing tissue dissection and mesh placement, but allows also easy and efficient treatment of the hernial orifices for seroma prophylaxis as part of robotic inguinal hernia treatment. In the following, the authors' standardised approach for the treatment of hernial orifices in robotic inguinal hernia treatment is described in more detail.

\section{Tailored approach}

In the authors' hospital, robotic inguinal hernia repair by rTAPP is the standard procedure; since May 2018, we have performed more than 600 rTAPP procedures. Complementary to the usual surgical steps, we make an additional effort to optimize the treatment of the hernial orifices in selected cases. In doing so, the hernias are classified intraoperatively in line with the EHS classification, according to the anatomic location $(\mathrm{L}=$ lateral; $\mathrm{M}=$ medial; $\mathrm{F}=$ femoral $)$ of the hernia and the size of the hernial orifices $(1=\leq 1$ finger; $2=1-2$ fingers; $3=\geq 3$ fingers) ${ }^{[9]}$. We focus on large medial orifices (EHS type M2 and M3) and lateral hernias with large hernia sac or large lipoma (EHS type L2 and 3) as well as inguinoscrotal hernias. Although waiving of mesh fixation has been shown to result in lower rates of postoperative seroma in the literature $\mathrm{e}^{[3]}$, it is also associated with an increased risk of recurrence, especially in large hernias. Therefore, in the author's series, mesh fixation is performed in all patients, although neither with glue nor with tacks, but with sutures. The robotic technology enables to perform mesh fixation with precise, superficially stitched, and loosely knotted absorbable sutures, without the risk of nerve damage. 


\section{Surgical treatment of the medial hernial orifice}

The medial hernial orifice is treated in large direct hernias (EHS M2 and M3) and/or if a considerable weakness of the transverse fascia exists. The fascia transversalis is progressively plicated or sutured to the iliopubic tract with a V-Loc suture; the suture is progressively performed concomitantly to the removal of the fatty tissue before the fascia is blown outwards by the pressure of the pneumoperitoneum. This approach takes some time ( $\sim-8 \mathrm{~min})$ due to the repetitive change of instruments (scissors/needle driver), but allows a very precise handling of the tissue, eliminating the risk of injury to the spermatic vessels, the cord, or nerve structures of the inguinal canal [Figures 1-3].

\section{Surgical treatment of the lateral hernial orifice}

In case of voluminous lateral hernias (EHS L2 and L3 with large hernia sac or lipoma) and in cases of scrotal hernias, the inguinal canal is sealed via the inner inguinal ring using fibrin glue spray (Tiseel, usually $4 \mathrm{~mL}$ ). For this purpose, a specific flexible cannula is available [Figures 4-6]. This step increases the operative time by approximately $3-5 \mathrm{~min}$. The application of the fibrin glue has to be performed by a scrubbed-in assistant familiar with the procedure.

\section{Mesh fixation}

In the authors' institution, mesh fixation during robotic rTAPP is performed in all cases. The robotic technology allows a minimally traumatic fixation of the mesh with four loosely knotted stitches with resorbable suture material (Vicryl 3-0). The location of the four sutures is as follows: (1) Cooper's ligament; (2) fascia of the rectal muscle; (3) fascia of the transverse muscle; and (4) iliac fascia [Figures 7-9]. Even the suture of the mesh to the iliac fascia in the location of the triangle of pain, can be very safely applied due to the excellent visual control helping to protect and exclude the nerves that are localized just below this fascia (i.e., N. cutaneous femoris lateralis and femoral and genital branches of the genitofemoral nerve) [Figure 8].

\section{CONCLUSION}

The treatment of the medial and lateral hernia orifice as part of endoscopic inguinal hernia treatment for postoperative seroma prophylaxis seems to be reasonable, especially in the case of large hernias. The use of DaVinci technology makes it thereby easier to apply the here presented techniques with utmost precision and accuracy.

The described 4-point suture fixation technique for the rTAPP with absorbable sutures is safe, according to the authors' experience. Skipping fixation with the inherent risk of mesh migration and hernia recurrence should not be advocated any more in times of robotic technology. A randomized evaluation of the described techniques in terms of postoperative seroma formation, chronic pain, or recurrence has yet to be performed. However, our preliminary, unpublished data concerning the strategies described above shows no elevated rates of chronic pain, no recurrence, and a clear decrease of the incidence of seroma. 


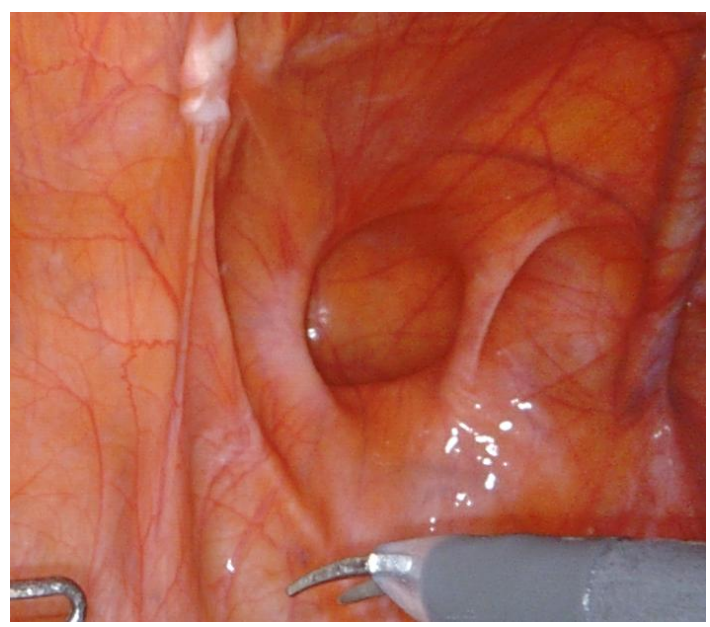

Figure 1. Initial situation with a right sided large direct hernia (EHS M3).

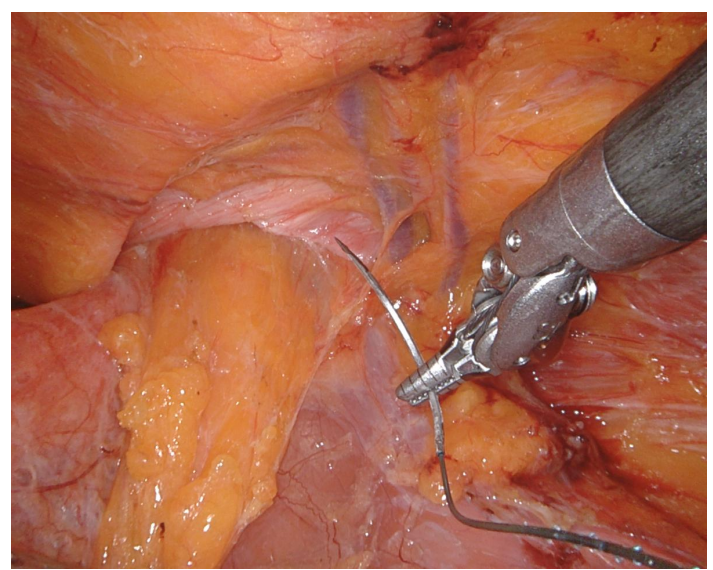

Figure 2. The suture of transverse fascia is performed step-by-step in alternation with the gradual mobilisation of the fatty tissue. In doing so, the surgeon can be sure that the suture does not damage vessels, nerves or the deferent duct, structures that are very close to the fascia transversalis. This prevents excessive protrusion of the transverse fascia into the groin during dissection (right side repair).

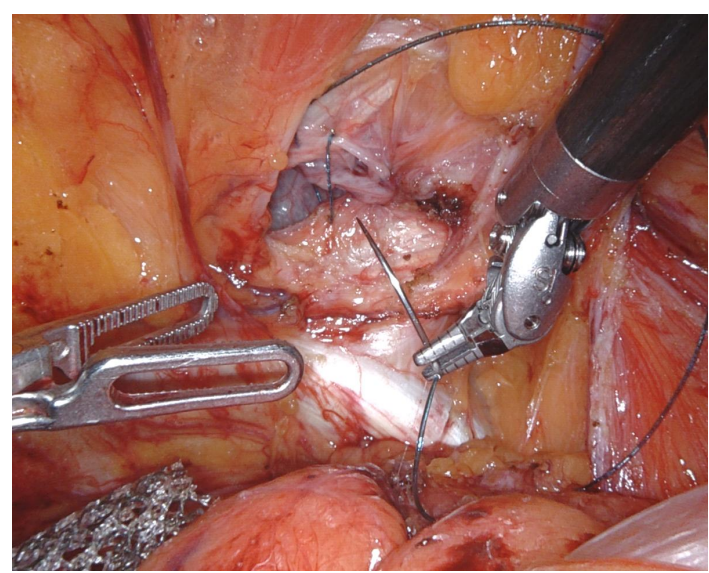

Figure 3. Suturing of the transverse fascia down to the iliopubic tract with a running $V$-Loc suture (right side repair). 


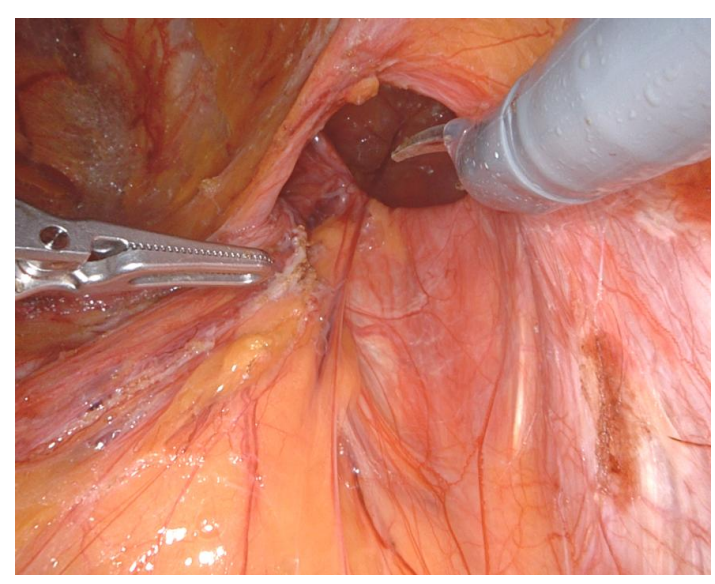

Figure 4. Lateral, right sided hernia with a large hollow space in the deep, due to the reduction of a respective lipoma and the outer sac. Prior to removal of the right instrument (scissors), it is positioned at the level of the inner ring, to facilitate later guidance of the fibrin glue application cannula by a correct angel of the trocar; this way, the $8 \mathrm{~mm}$ DaVinci port remains connected to the respective DaVinci arm.

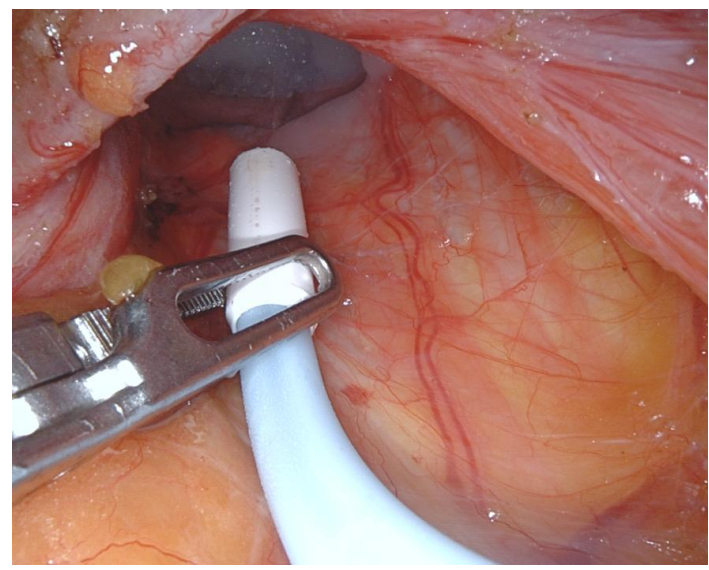

Figure 5. Endoscopic view into the inguinal canal with the Tisseel applicator already in place. The fibrin application cannula is guided by the Prograsp forceps. With the wrist movements of the Prograsp, the fibrin glue can be sprayed in all directions, covering the whole inner surface of the tissues of the inguinal canal (right side repair).

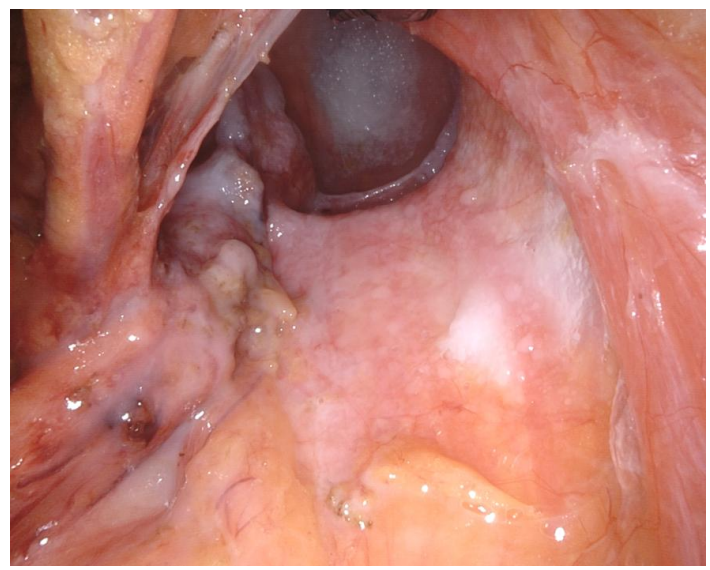

Figure 6. Inguinal canal lined and sealed with fibrin glue spray. With the $30^{\circ}$ optic the result can be visualised deep into the hollow space (right side repair). 


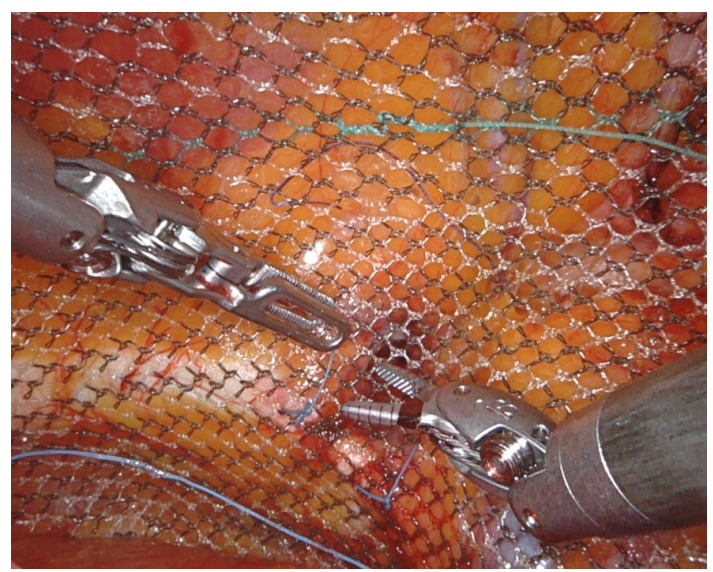

Figure 7. Mediocaudal fixation of the mesh to Cooper's ligament (ligamentum pectinatum) (right side repair).

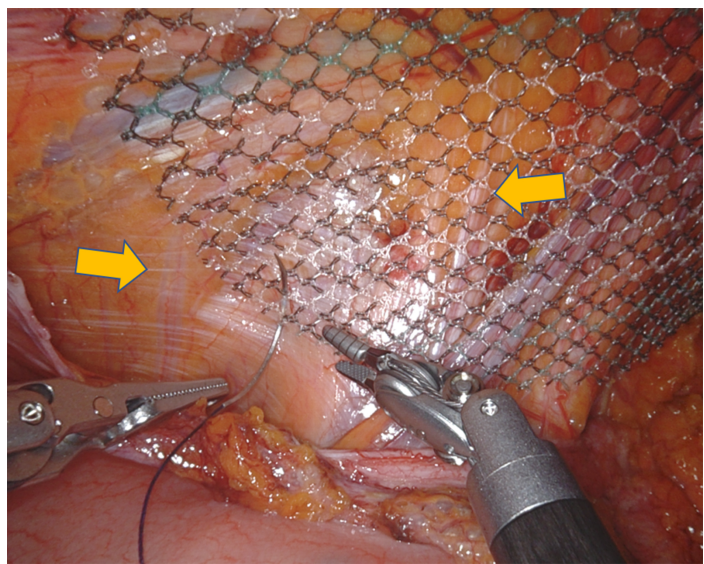

Figure 8. Laterodorsal fixation (left side repair). The fixation is done by a very superficially stitched suture including only the iliac fascia, with safe exclusion of the clearly visible nerves (yellow arrows) of the lateral abdominal wall.

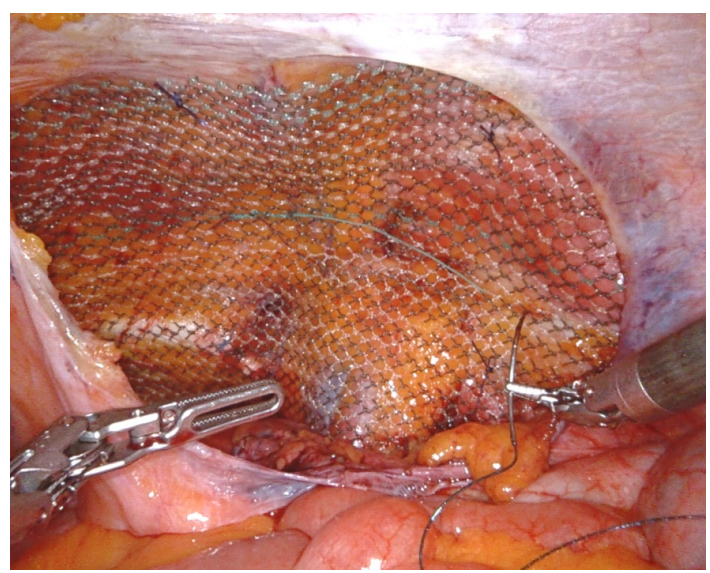

Figure 9. Mesh fixed with 4 single sutures (right side repair). 


\section{DECLARATIONS}

\section{Authors' contributions}

Collecting imaging material and manuscript writing: Baur J, Ramser M, Dietz UA

\section{Availability of data and materials}

Not applicable.

\section{Financial support and sponsorship}

None.

\section{Conflicts of interest}

Dietz UA is proctor for Intuitive. All other authors declared that there are no conflicts of interest.

\section{Ethical approval and consent to participate}

The scientific analysis of patient data in the authors' clinic is covered by an ethical vote of the University of Basel (EKZN 2019-02046). Patients have consented to the use and publication of their medical data collected in the course of medical care for scientific purposes.

\section{Consent for publication}

Patients have consented to the use and publication of their medical data collected in the course of medical care for scientific purposes.

\section{Copyright}

(c) The Author(s) 2021.

\section{REFERENCES}

1. Manangi M, Shivashankar S, Vijayakumar A. Chronic pain after inguinal hernia repair. Int Sch Res Notices 2014;2014:839681. DOI PubMed PMC

2. Group. International guidelines for groin hernia management. Hernia 2018;22:1-165. DOI PubMed PMC

3. Köckerling F, Bittner R, Adolf D, et al. Seroma following transabdominal preperitoneal patch plasty (TAPP): incidence, risk factors, and preventive measures. Surg Endosc 2018;32:2222-31. DOI PubMed PMC

4. Li J, Gong W, Liu Q. Intraoperative adjunctive techniques to reduce seroma formation in laparoscopic inguinal hernioplasty: a systematic review. Hernia 2019;23:723-31. DOI PubMed

5. Berney CR. The Endoloop technique for the primary closure of direct inguinal hernia defect during the endoscopic totally extraperitoneal approach. Hernia 2012;16:301-5. DOI PubMed

6. Li J, Zhang W. Closure of a direct inguinal hernia defect in laparoscopic repair with barbed suture: a simple method to prevent seroma formation? Surg Endosc 2018;32:1082-6. DOI PubMed

7. Sürgit Ö, Çavuşoğlu NT, Kılıç MÖ, Ünal Y, Koşar PN, İçen D. Use of fibrin glue in preventing pseudorecurrence after laparoscopic total extraperitoneal repair of large indirect inguinal hernia. Ann Surg Treat Res 2016;91:127-32. DOI PubMed PMC

8. Pini R, Mongelli F, Proietti F, et al. Suture and Fixation of the Transversalis Fascia during Robotic-Assisted Transabdominal Preperitoneal Hernia Repair to Prevent Seroma Formation after Direct Inguinal Hernia Repair. Surg Innov 2020:1553350620960976. DOI PubMed

9. Miserez M, Alexandre JH, Campanelli G, et al. The European hernia society groin hernia classification: simple and easy to remember. Hernia 2007;11:113-6. DOI PubMed 